\title{
Review of Expanding Program on Immunization (EPI) in Sudan 2018
}

\author{
Suliman Abaker* \\ Department of Communicable Diseases Control, Karary University, Sudan \\ *Corresponding Author: Suliman Abaker, Department of Communicable Diseases \\ Control, Karary University, Sudan.
}

DOI: 10.31080/ASMI.2020.03.0520
Received: January 06, 2020

Published: February 10, 2020

(c) All rights are reserved by Suliman Abaker.

\begin{abstract}
Immunization is one of the most successful global health interventions and cost-effective ways to save lives and prevent diseases. Sudan is the third largest country in Africa, with a land area of 1.882 million km2. It shares borders with seven countries and has a coastal line along the Red Sea.The EPI program in Sudan is the most successful primary health care intervention in the country. Immunization in Sudan is by law mandatory to all target populations and vaccination cards are mandatory for school entry. Vaccines are provided free of charge to all target population living in the country regardless of their nationality, including the refugees.

Routine immunization program in Sudan has good political support which has been translated into modest financial support. The routine immunization coverage in the country varies with different antigens; there are vaccines that have achieved the targeted coverage of more than 90\% since 2008 (BCG, Penta3 and polio3) with dropout rate (DOR) between first and third dose more than $5 \%$. The year 2017 witnessed great improvement in measles vaccination that was stagnant for several years, where MCV1reached $90 \%$ for the first time and MCV2 reached $72 \%$ at national level.
\end{abstract}

Keywords: WHO; EPI; GAVI; Sudan; UNICEF; Immunization; Vaccine

\section{Background}

Immunizations are estimated to prevent more than two million deaths a year globally. It is one of the most successful global health interventions and cost-effective ways to save lives and prevent diseases [1].

The World Health Organization (WHO) initiated the Expanded Program on Immunization (EPI) in May 1974 with the objective to vaccinate children throughout the world. After ten years, in 1984, the WHO established a standardized vaccination schedule for the original EPI vaccines which include Bacillus Calmette-Guerin (BCG), Diphtheria-tetanus-pertussis (DTP), oral polio, and measles. Increase knowledge of the immunologic factors of disease led to new vaccines being developed and added to the EPI's list of recommended vaccines like Hepatitis B Virus (HBV), yellow fever in countries endemic for the disease, and Hemophilus influenzae meningitis (Hib) conjugate vaccine in countries with high burden of the disease [5].

All the national governments of the member states the United Nation create and implement their policies for vaccination programs following the guidelines set by the EPI. Setting up an immunization program is multifaceted and contains many complex components including a reliable cold chain system, transport for delivery of the vaccines, maintenance of vaccines stocks, training and monitoring of health workers, outreach educational programs to inform the public, and a means of documenting and recording which child receives which vaccines [6].

In 1999, the Global Alliance for Vaccines and Immunization (GAVI) was created with the sole purpose of improving child health in the poorest countries by extending the reach of the EPI. The GAVI brought together a grand coalition, including the UN agencies and institutions (WHO, UNICEF, and the World Bank), public health institutes, donor and implementing countries, the Bill and Melinda Gates Foundation and the Rockefeller Foundation, the vaccine industry, non-governmental organizations (NGOs) and many more. The creation of the GAVI has helped to renew interest and maintain the importance of immunization in battling the world's large burden of infectious diseases [5].

\section{The republic of Sudan}

Sudan is the third largest country in Africa, with a land area of 1.882 million $\mathrm{km}^{2}$. It shares borders with seven countries and has a coastal line along the Red Sea. Sudan is a multiracial, multicultural nation distributed along 18 states and 189 localities only four of them lies South Kordofan and Blue Nile states, which completely closed because of conflict. The total population accounts to $40,782,742$ Million people out of which around $70 \%$ live in rural areas and $8 \%$ are pastoralists. There are 2.2 million internally dis- 
placed people, and refugees from neighboring countries amount to another 2 million about $74 \%$ of South Sudan refugees out of camps [1].

Large refugee populations are found in Algeria, Egypt, Iraq and the Sudan. These Governments are stretched to provide health and immunization services to these populations and adapt delivery strategies to reach displaced persons. Nomads, recent urban migrants, certain ethnic groups and people living in more remote geographic areas, in addition to refugees, have less access to immunization services [11].

The significant population movement and influx of refugees, Internal Displaced Population (IDP) and returnees: Instability in neighboring countries and in the region resulted in high number of refugees who crossed the boarders to Sudan [3].

Sudan faces economic crises and impacts of the sanction for more than 20 years results in increase of inflation rate, devaluation of the local currency and severe shortage of fuel. The impact of economic situation on health includes disruption of provision of health services and delays in implementation of some interventions including, immunization campaigns [7].

Access to conflict affected areas and access to health services have improved in areas that were not accessible before, mainly in Jabal Marra in Darfur region. However, some part of Jabal Marra (upper mountain villages), in addition to the three areas in South Kordofan and Blue Nile States are still inaccessible. More advocacy and involvement of tribal and community leaders is ongoing to ensure that population in these areas will have access to basic health services including immunization [7].

\section{The national EPI program in Sudan}

The EPI program in Sudan is the most successful primary health care intervention in the country. Immunization in Sudan is by law mandatory to all target populations and vaccination cards are mandatory for school entry. Vaccines are provided free of charge to all target population living in the country regardless of their nationality, including the refugees [1].

EPI program was launched in Sudan in 1976. The first fiveyear plan for the EPI was formulated in 1985. The program had introduced the six classical EPI antigens (BCG, polio, DPT, measles). In 1990, vaccination coverage of children under one tear of age reached $62 \%$ for DPT3 as a national figure. During the period 1990 - 1994, EPI coverage dropped to 51\% due to lack of financial and material support from the government of Sudan and from donors. During the period 1995-2001was not sustained and ranged between 50 and $79 \%$. Sudan has since strengthened its routine EPI activities with some success: increased coverage rates, successful
NIDs, strengthened disease surveillance with emphasis on AFP surveillance and improvement of the cold chain capacity and quality. In early 2005, with funding from GAVI, the EPI introduced Hepatitis $B$ Virus (HBV) vaccine in three states with extension to six states by the end of 2005 and with a national roll out planned for 2006. Polio, measles and MNT campaigns supplement the routine programs [3].

In the year 2011 Sudan has witnessed the introduction of Rota virus vaccine in Sudan, the first country in Africa to introduce Rota vaccine with support from the GAVI Alliance. Rota vaccine addresses severe diarrhoeal disease in infants and young children caused by Rotavirus [2].

EPI is one of the Primary Health Care (PHC) departments at the FMoH. At the federal level, all policies, technical guidelines and plans are developed in collaboration with the states and then disseminated to all levels. This level is also responsible for training, technical support, supervision, monitoring and evaluation of the states service performance. The federal level is managed by the National EPI Director together with the heads of the different EPI sections: Surveillance, SIAs, Immunization Safety, Information and Research, Social Mobilization, Training, Cold Chain Management, GAVI, and Operations [3].

\section{EPI support in Sudan}

The EPI program largely depends financially on international partners who cover almost all vaccines required by the program (the Government of Sudan co-finances the new vaccines) and a most of the vaccine delivery operational costs for both routine schedules and campaigns [2].

Sustainable financial support has been provided by GAVI and other partners like the Polio Eradication Initiative, measles elimination Initiative, the UNICEF and WHO. GAVI support has been used primarily to improve routine immunization delivery, with a focus on establishing strong outreach and mobile strategies, recruiting and training vaccinators and providing incentives to staff to implement the outreach and mobile activities; and improving the cold chain and vaccine management. GAVI funds also cover vaccines and operational costs for yellow fever and meningococcal A vaccination campaigns. The polio and measles initiatives have been providing substantial support to help the country implement polio eradication and measles elimination strategies respectively. All vaccines required by the program are procured through UNICEF Supply Division [10].

Routine immunization program in Sudan has good political support which has been translated into modest financial support. Hence the establishment of post marketing surveillance of intussusceptions and Government co-sharing of the cost of new vaccines 
has been successfully carried out across the biennium. In addition, GAVI Alliance approved to support introduction of meningococcal A conjugate vaccine in 2012 - 2013. This is part of the massive introduction of the vaccine in 25 countries comprising the African meningitis belt, with the ultimate goal of eliminating the disease from Africa [2].

\section{Cold chain and vaccine management system in Sudan}

Sudan has put in place a strong cold chain and vaccine management system that covers all administrative levels and secures continuous availability of required vaccines to all vaccination points in the country, including in the remote and high-risk areas. Sudan was the first country in the world to be certified by UNICEF for its excellent vaccine management system. The nationwide meningococcal A conjugate vaccine (Men A) and yellow fever vaccination campaigns in 2012 - 2013 and 2014 - 2015 respectively confirmed the high performance of the system [1].

Immunization services in Sudan delivered through three main strategies; fixed, outreach and mobile. The EPI target is to expand the fixed services to cover most of the population, as the other two strategies are more expensive a success has been achieved, however more effort will be done in the coming years utilizing the opportunity of PHC expansion as well as the CCE OP support to open more fixed sites [2].

A strong vaccine preventable diseases surveillance system has been put in place and the program has reached the recommended performance standards, in particular for acute flaccid paralysis (AFP), and fever and rash laboratory case-based surveillance, as confirmed by independent reviews and assessments. The program operates through a national multi-year plan (2001 - 2016), translated annually into national and state action plans as well as district micro-plans. A new multi-year plan, covering 2017 - 2021, will develop based on the Global and Regional Vaccine Action Plans $[1]$.

\section{EPI coverage in Sudan}

Each year WHO and UNICEF jointly review reports submitted by Member State regarding national immunization coverage, finalized survey reports as well as data from the published and grey literature. Based on these data, with due consideration to potential biases and reviews of local experts, WHO and UNICEF attempt to distinguish between situations where the available empirical data accurately reflect immunization system performance and those where the data are likely to be compromised and present a misleading view of immunization coverage while jointly estimating the most likely coverage level for each country [4].

The population used for vaccine need calculation was based in the EPI administrative population data estimation for 2017. This operational figure provide opportunity for more accurate and reliable vaccines and other immunization supplies forecasting. The program has reached all the planned targeted coverage with slight increase for Pentavalent (94.7\%), Rota (95.2) and PCV (94.6\%). Inactivated Polio Vaccine (IPV) was not available (stock out) throughout the year due to global vaccine shortage. Vaccine wastage rate for GAVI-supported and traditional vaccine for antigens is within the recommended wastage rate as was reported during 2017 [7].

Implementation of Reaching Every District (RED) approach facilitated equitable in immunization services. Vision of EPI in Sudan is to reach every child regardless of his or her sex, ethnicity, tribe, location, rich or poor [8].

The routine immunization coverage in the country varies with different antigens; there are vaccines that have achieved the targeted coverage of more than $90 \%$ since 2008 (BCG, Penta3 and polio3) with dropout rate (DOR) between first and third dose more than 5\%. The year 2017 witnessed great improvement in measles vaccination that was stagnant for several years, where MCV1reached $90 \%$ for the first time and MCV2 reached $72 \%$ at national level [7].

Access for vaccination teams in the areas of armed conflict is difficult and risky but the continued cooperative efforts between the government, armed groups, local communities, nongovernmental organization, United Nations agencies and the African Union/UN Hybrid operation in Darfur (UNAMID) make exceptions to the rules of war. Vaccination reams enter the area under military protection, immunize the children and leave as soon as possible. The government has also allowed volunteers from the armed-conflict areas to travel to government-controlled areas for training, providing them with vaccines and other support so that they can go back and vaccinate the children in areas where it is too risky to send in a team of outsiders [12].

Sudan adopt several approaches to enhance equitable service delivery and reaching every and last child. These approaches are: Implementation of systems to identify and reduce missed opportunities to vaccination, Second year vaccination and booster vaccination, and Full implementation of Reaching Every Community approach (REC) [9].

Unfortunately, in the years 2015, 2016 the program has been facing difficulties to sustain the outreach and mobile strategies, mainly because of insufficient financial resources, lack of transportation means, high staff turn-over, and lack of training, these challenges negatively affect the program and make delays in vaccine distribution, but fortunately, in the binging of the year 2017 these challenges has been solved [1]. 


\section{Polio eradication program}

Sudan was one of the countries that adopted WHA resolution to eradicate poliomyelitis by the year 2000. Eradication efforts started in 1994 triggered by occurrence of a major polio outbreak. The conducted activities resulted in eradication of the endemic virus in the country and occurrence of a high quality AFP surveillance and OPV vaccination coverage. The first importation in $2004-2005$ from Chad causes 158 polio cases in 18 States of the country. The importations in 2007, 2008 and 2009 caused limited outbreaks. The last polio case reported in the country was from Port Sudan, Red Sea State in the $15^{\text {th }}$ of March 2009 [13].

\section{Conclusion}

The national EPI program in Sudan is the most successful primary health care intervention in the country. Vaccines are provided free of charge to all target populations living in the country regardless of their nationality, including the refugees. The international partners cover all vaccines required by the program, while the government cover the new vaccines co-financing and most of the vaccine delivery operational costs. Substantial financial support has been provided by many partners like GAVI, The Polio Eradication Initiative, the measles elimination Initiative, UNICEF, WHO and others.

\section{Bibliography}

1. Joint External Evaluation of IHR Core Capacities of the Republic of the Sudan. Geneva: World Health Organization (2017).

2. Republic of the Sudan, Federal Ministry of Health, Expanded Program on Immunization: Annual Report (2017).

3. Republic of the Sudan, Federal Ministry of Health, Expanded Program on Immunization: Comprehensive Multi-Year National Immunization Plan (2010).

4. Sudan: WHO and UNICEF estimates of immunization coverage (2016).

5. Jamison D., et al. "Disease Control Priorities in Developing Countries, Second Edition?". 2006, The World Back Group Archived (2013).

6. Hadler S., et al. "Vaccination Program in Developing Countries". Chapter 55: Vaccines (2004).

7. GAVI Sudan Joint Appraisal report (2018).

8. The GAVI Alliance: Global Alliance for Vaccines amd immunization (2017).
9. Ismail IT., et al. "Assessment of Routine Immunization Coverage in Nyala Locality, Reasons behind Incomplete Immunization in South Darfor State, Sudan". Asian Journal of Medical Sciences 6.1 (2014): 1-8.

10. UNICEF - Sudan: Annual report (2017).

11. UNICEF: Immunization Financing in MENA Middle-Income Countries (2018).

12. WHO: Strengthening routine immunization in Sudan (2013).

13. Republic of Sudan, Federal Ministry of Health, Expanded Program on Immunization: Sudan EPI Sudan Benefits from Polio Eradication Program - Polio Legacy planning and Implementation Workshop - Geneva (2015).

\section{Assets from publication with us}

- Prompt Acknowledgement after receiving the article

- Thorough Double blinded peer review

- Rapid Publication

- Issue of Publication Certificate

- High visibility of your Published work

Website: https://www.actascientific.com/

Submit Article: https://www.actascientific.com/submission.php Email us: editor@actascientific.com

Contact us: +919182824667 